\title{
Should injury prevention programmes be targeted
}

\author{
Heather Ward
}

The argument is spurious whether to distinguish between injury prevention strategies aimed at the population as a whole or at high risk individuals. Both are required as injury prevention across all types of accidental injury needs to be managed strategically and to be community based. Most community based strategies will encompass the needs of different groups and will be effective in reducing injuries across the community as a whole. Also we know quite a bit about population based strategies and relatively little about high risk groups in the context of injury prevention.

\section{What is risk?}

There is confusion as to what risk actually means and this confusion is muddying the waters about what to do about reducing it.

The risk of an incident occurring which results in injury depends on the opportunity for such an incident to happen; this opportunity is commonly called exposure. The likelihood of such an incident occurring, given the opportunity, is risk. Risk is usually expressed as an injury rate relative to an amount of exposure such as pedestrian injuries per 100 million roads crossed. This gives an objective measure of risk which can be attached to a location, such as a stretch of road or junction, or to an activity such as a car journey or a walk to the shops. This measure is independent of the individual but can relate to subpopulations such as children, older people, males or females. In the absence of exposure data the fallback position has been to use per capita information so we see risk associated with injuries per 100000 population of that age group, gender, or socioeconomic group. This is an approximation where population is a proxy for exposure but should be used with caution. A study by Ward et al of pedestrian activity and accident risk showed that, on average, females walk more and cross more roads than do males but are generally less at risk of injury as pedestrians. ${ }^{1}$ The risk of injury changes with age and this has great importance for policy decisions about appropriateness of differing types of interventions.

Another use of the word risk is perceived risk. An example of this is where people find a location or activity so threatening that they avoid it thus reducing their likelihood of being casualty records. Fortunately professionals are now beginning to understand more about this mismatch and are taking steps to reduces danger as perceived by the community.

A third use of risk relates to the behaviouf of individuals in terms of risk taking. Whiles high risk behaviour does not often result in accidental injury there is evidence to support the premise there are relationships for indivi- 8 duals over a medium to long period of time? Work being carried out by West on driving deviance is indicating that the top $25 \%$ of scorers on his attitude to driving deviance questionnaire had two to three times thes accident rate on average of those in the्ष bottom quartile. ${ }^{2}$ Drivers who in the past two years have had an accident for which theye could be held at least partly responsible have four times the accident risk of a similar accident in the following year. Even in studiess of individual differences in accident risk exposure, and environmental factors are helof constant in the analysis.

\section{How relevant are social factors?}

What about social factors? Christie in her. work on the high risk child pedestrian looked. at socioeconomic and environmental factors in their accidents. ${ }^{3}$ The casualty and socios demographic data indicate that children from? low income families are much more likely to be injured as pedestrians compared with children of the same age and sex in high: income groups. Christie found evidence that children from the lower socioeconomic groupso are more exposed to traffic compared with higher groups especially in the context of using the street for recreation, which is often unsupervised by adults. Another importani․ finding was that some parents may feel less able to safeguard their children in traffic and particularly in the case of ethnic groups, are less informed about traffic risk. Too little is known about the high injury involvement of low income groups, what the interplay is of social and urban deprivation, and how much personal variables influence exposure to certain situations.

Even with low income groups the propensity

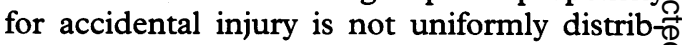
uted among the subpopulation nor, justo because the group is low income, does it mean that it will have a high accident rate. The othero problem with prevention of accidental injury is that we do not know who is going to be injuredô nor where. Hence the dilemma as whether to target individuals, small groups, or the whole population.

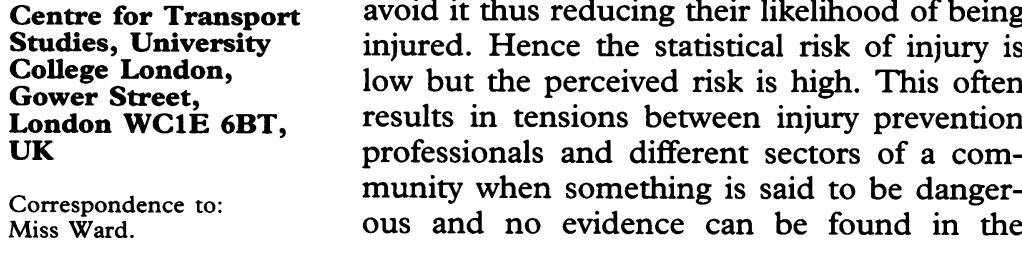

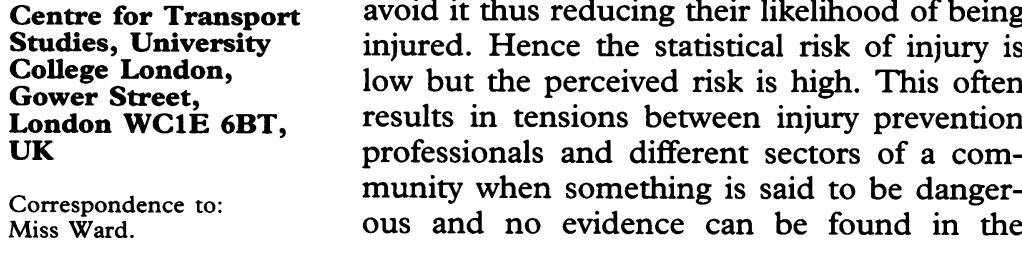

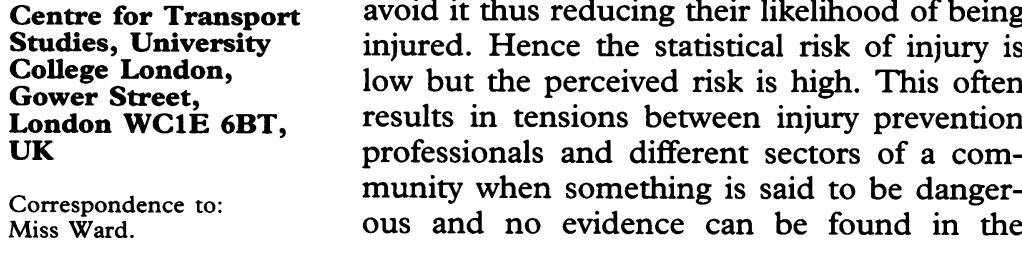

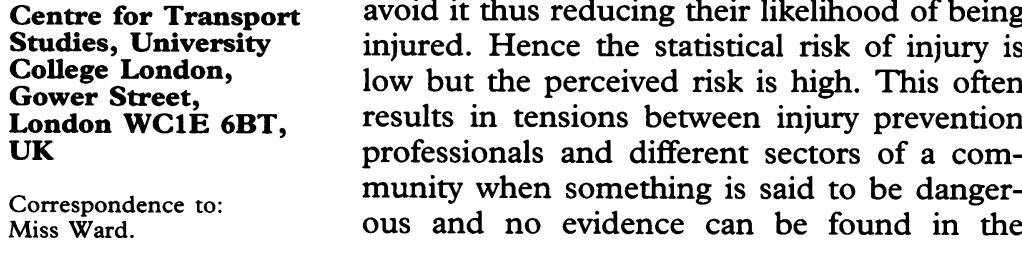

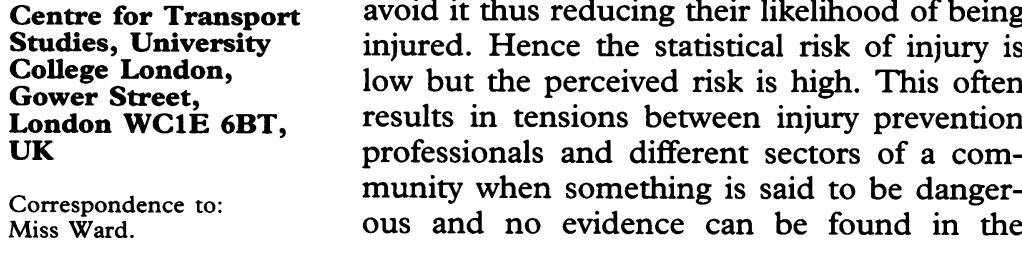

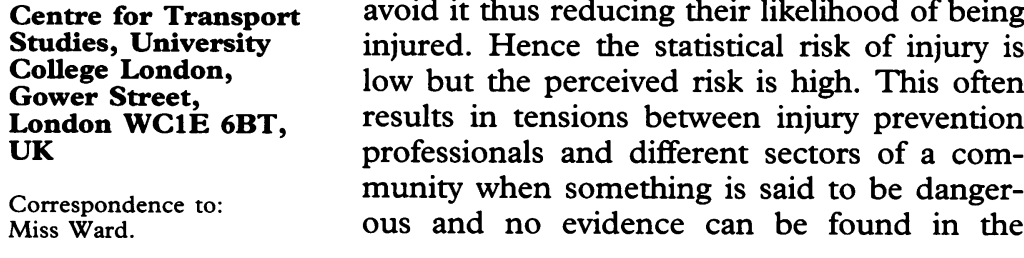




\section{THE CONCEPT OF RELATIVE RISK}

What is needed is more work to estimate relative risks of various activities undertaken in different locations and to different groups of individuals.

Some population, or community based interventions have been highly successful. Stair gates, child resistant tops for medicines and poisons, and enclosures for private swimming pools are three examples. These have succeeded because people have understood the real risks associated with falls down stairs, poisoning, and drowning. It is suspected that many other interventions have not worked as well as may have been expected simply because the likelihood of an event happening to a given individual is very small and, therefore, the risk is perceived to be low with the consequence that behaviour is not modified accordingly. Road traffic accidents that result in injury are rare events which, thankfully, are not experienced by many people, leading them to underestimate the risk to themselves. The events leading up to an accidental injury are complex as there is no single cause. An example of this might be where a mother takes her child by car to the supermarket nearby; she does not fasten the child seat restraint because it is a fiddle and as it is raining she will get wet and, anyway, it is not far to go. On the way, a van pulls out in front of her. As it is raining she cannot stop in time, a collision occurs and the child, being unrestrained, is injured. The risk of injury is not related to the distance from home, it is rather the reverse as there are more short journeys made than long ones and each journey has a risk associated with it.

Most parents are able to determine for themselves the risk of their child falling down ungated stairs whereas they have totally inadequate information to be able to do this in the context of a road traffic accident leading to over or under assessing the risks. Why should this be? The exposure to the risk of injury from falling down stairs is high because the young child spends the majority of its time in the house where the opportunity to fall down is relatively high. Relatively few children are injured in the road environment and when they are it is usually close to home because that is where they spend most of their time. Notwithstanding this, the risk of crossing a main road close to home is greater than crossing a residential road at the same distance from home. This may not be intuitive to a parent and more information needs to be disseminated on these relative risks. The message that a child is not a miniature adult needs to be addressed to all parents but it is the style of the message which needs to change according to the home situation.

Attitudes, motivations, social norms, and perceptions determine how people behave in a given situation. Certain attitudes may have been formed from erroneous or incomplete information. Understanding how attitudes are formed is therefore an important first step before devising ways of changing them to effect changes in behaviour. Wilde comments on this as follows ${ }^{4}$ : $\therefore$. . accident rate and the incidence of unhealthy habits essentially depend on people's orientation towards their future. The more they expect from $i t$, the more careful they will be with life and limb. If their expectations are low, they will try to find more immediate gratification of their desires, and do so at greater risk of jeopardising their lives. The extent of risk taking with respect to safety and health in a given society, therefore, ultimately depends on values that prevail in that society, and not on the available technology'.

\section{The power of information}

Here we turn to the importance of society in the widest sense and the community in the local sense. For interventions to be successful, they must have the consent of the target group as well as the consent of society or the community. In addition, the interventions must address the cause of the problem which often requires consensus among different groups of professionals. Much injury prevention work is undertaken in a piecemeal fashion with different professional groups having an interest in a particular type of injury. This is counterproductive as many of the underlying factors that increase risk of injury are societal or environmental. The detailed solutions to the particular injury patterns may be different because some may be targeted at reducing exposure to the risk such as stair gates, child proof containers, safe electrical wiring and some may be directed at modifying behaviour to reduce risk such as training people to use equipment in the proper manner or to cross the road or drive a car. Both are effective strategies for small target groups and for the population as a whole.

Some successful road safety interventions have these necessary ingredients and have been demonstrated to reduce injuries. Here communities work with professionals to understand the road safety problems they face and together devise solutions that are acceptable to the majority. The solutions include road engineering, school based projects, health visitor projects, and enforcement strategies all aimed at bringing together more closely the perceived and actual risks, thus giving the community sufficient information on which to act to solve its own road safety problems with the help of the professionals. Some of the projects will target specific accident types or groups of people within the community, others will be directed at the whole community, helping it to understand and manage risks in ways that are acceptable to itself. This will vary from community to community as social and demographic profiles differ even between communities that are geographically adjacent.

The main mechanisms for this strategy, which enables individuals to understand and manage their own risks, are the provision of information, reinforcement through encouragement, and physical change to the environment, all backed up by legislation where appropriate. However, within this wider approach there will remain groups which form the tails of the various injury distributions, or 
whose behaviour, through being sufficiently deviant, poses a risk to others.

For a planned intervention to be effective, the people in these groups, or their carers, will need sufficient information to understand the need for, and to agree to, any action. The challenge for professionals is to communicate the process of implementation of specially targeted interventions designed to reduce the risk of injury to themselves or to others in the community.
1 Ward $\mathrm{H}$, Cave J, Morrison A, et al. Pedestrian activity and accident risk. Basingstoke: AA Foundation for Road Safety Research, 1994.

2 West RJ. Individual differences in accident risk: a review of findings and an examination of methods. Behavioura $\bar{D}$ research in road safety VI. Proceedings of a seminar at Royal Holloway College, University of London, 4-5 September 1995. PR3138/96. Crowthorne: Transpore Research Laboratory, 1996.

3 Christie N. The high risk pedestrian: socio-economic and environmental factors in their accidents. Project Reporf? Transport Research Laboratory, 19950 4 Wilde GJS. Target risk. Toronto: PDE Publications, 1994.

\title{
Population strategies for prevention? If only it were that simple!
}

\author{
Jerry Moller
}

The paper by Kendrick and Marsh (p170) sparked this Dissent. On the basis of this one study, in one general practice in the UK, the authors suggest that injury prevention targeted at groups at higher risk is not worthwhile and that a universal approach to injury prevention is preferable. I disagree, despite the thoughtful Opinion of Ward (p160) who defends this conclusion.

The debate about universal (or population based) versus high risk strategies has ebbed and flowed for 20 years. It was a central issue in arguments about the welfare state, ${ }^{1}$ and reappeared in suggestions that indicated that health promotion has contributed to increasing differentials between rich and poor by adopting a universal approach. Little has been written about this issue with respect to injury, but as Kendrick and Marsh point out, many recommendations have been made for targeting high risk groups, especially poor children, based on injury rate differentials. The lack of debate has created a climate where the responses of policy makers and researchers are often over simplified.

We must first consider why the leap from a single study to the conclusion that universal strategies are the best approach is simplistic, despite Ward's support. The Kendrick and Marsh paper considers only some of the layers of complexity and argues that universal approaches may increase the rich-poor differential. Targeted strategies are necessary, the authors say, if this disadvantage is to be avoided.

\section{Injury Information Services, Australian Institute of Health and Welfare, National Injury Surveillance Unit, Adelaide, South Australia}

Correspondence to: Mr J Moller, PO Box 38, Mt Compass, SA 5210 , Australia.

\section{Measuring risk}

The measurement of risk is itself, a complex issue. How does the risk of individuals, or communities relate to population risk? At what level of severity should risk be measured? There may be little difference in risk measured across the full range of severity ranging from first aid to medical treatment to death, but large differentials among the most severe injuries, such as those resulting in long term disability or death. One subpopulation, indi- $\frac{\mathbb{D}}{\mathbb{O}}$ cated by broad measures to be at higher risk, may not have a measurable difference in injury rates in a short term or cross sectional study $\overrightarrow{x 0}$ purely because of random variations in injury incidence. Differences may also be hidden by inappropriate use of age standardization or byo failure to consider maturation effects.

It should be remembered that the indicatorso of high risk are often relatively insensitive proxyळ measures. Jolly et al have shown that composite measures of socioeconomic status underesti-O mate the relationship between poverty and injury rates because components of socio economic status, such as education and income, correlate in opposite directions with injury rates under some circumstances. ${ }^{2}$

In the light of this, it is not surprising that some studies will show a strong relationship between poverty and risk while others show no such relationship. Equally, just as it is falla-? cious to believe that every individual in a high risk group has an elevated risk of injury, it is also a fallacy to believe that every under- $\frac{0}{3}$ privileged community will experience elevated injury rates.

\section{Trends in injury patterns for the population}

Cross sectional and short term studies easily lead to misunderstandings about theo need for targeted interventions. Consider figo 1: a small difference in injury rate occurs at the beginning; it increases over time, but the? rates of injury drop for both groups. The difference is insufficient to be seen to justify $a$ 응 targeted intervention and a universal interven- $-\stackrel{\Phi}{\Omega}$ tion is commenced. It has a larger effect on $\stackrel{\mathbb{Q}}{\AA}$ the low risk group than the high risk group The differential is increased, even thougho both groups have benefited. Failure to identifyo the need to undertake a targeted intervention has resulted in an increased differential. The difference may have become more entrenched and the cost of intervening may have increased. 